\title{
Aliette Geistdoerfer, le CETMA-Anthropologie maritime et son centre de documentation
}

Aliette Geistdoerfer, CETMA-Anthropologie maritime and its resource centre

\section{Émilie Mariat-Roy et Christophe Sécula}

\section{(2) OpenEdition}

\section{Journals}

Édition électronique

URL : http://journals.openedition.org/ethnoecologie/3529

DOI : $10.4000 /$ ethnoecologie.3529

ISSN : 2267-2419

Éditeur

Laboratoire Eco-anthropologie et Ethnobiologie

Référence électronique

Émilie Mariat-Roy et Christophe Sécula, « Aliette Geistdoerfer, le CETMA-Anthropologie maritime et son centre de documentation », Revue d'ethnoécologie [En ligne], 13 | 2018, mis en ligne le 25 juin 2018, consulté le 05 mai 2019. URL : http://journals.openedition.org/ethnoecologie/3529 ; DOI : 10.4000/ ethnoecologie.3529

Ce document a été généré automatiquement le 5 mai 2019.

Revue d'ethnoécologie est mis à disposition selon les termes de la licence Creative Commons Attribution - Pas d'Utilisation Commerciale - Pas de Modification 4.0 International. 


\title{
Aliette Geistdoerfer, le CETMA- Anthropologie maritime et son centre de documentation
}

Aliette Geistdoerfer, CETMA-Anthropologie maritime and its resource centre

\author{
Émilie Mariat-Roy et Christophe Sécula
}

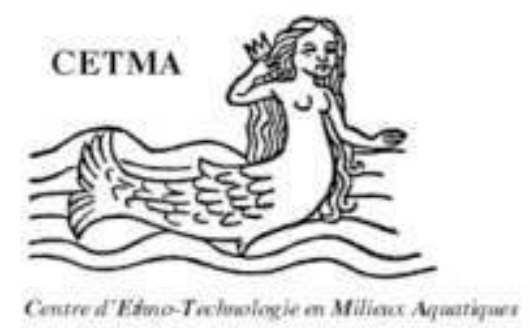

1 Aliette Geistdoerfer (1943-2015) est la fondatrice de l'anthropologie maritime en France. Son travail de recherche est inséparable de celui de la fondation du Centre d'EthnoTechnologie en Milieux Aquatiques (CETMA) ${ }^{1}$ et de sa bibliothèque.

2 Centre de documentation unique en sciences humaines et sociales à caractère pluridisciplinaire et association Loi 1901, le CETMA-Anthropologie maritime s'inscrit dans les recherches engagées au Muséum national d'histoire naturelle sur les formes d'appropriation sociale de la nature, plus particulièrement celle des mers et océans.

Dès 1969, à son retour des îles de la Madeleine (Canada), Aliette Geistdoerfer rejoignit le Laboratoire d'Ethnobotanique du Muséum alors dirigé par le Professeur Portères, d'abord comme vacataire puis comme Chargée de recherches du $\mathrm{CNRS}^{2}$ où elle fut nommée en 1973. Parallèlement, elle s'engageait dans une thèse de doctorat d'Etat sous la direction du Professeur Leroi-Gourhan ${ }^{3}$.

4 Grande chercheuse de terrain, Aliette était spécialiste des communautés de marinspêcheurs de l'Atlantique nord (îles de la Madeleine, Saint-Pierre-et-Miquelon, Bretagne, etc.). Elle a consacré ses recherches aux populations de marins-pêcheurs, à leur organisation sociale et à leurs pratiques d'exploitation des océans. Elle s'est beaucoup 
attachée, aussi, aux rôles et places qu'occupent les femmes dans l'organisation sociale des communautés maritimes.

5 Nommée Directeur de recherches du CNRS, Aliette y a dirigé l'UMR 5196 Techniques et Culture et fut responsable des RCP «Anthropologie maritime». Membre associé de l'équipe de recherche APSONAT (Appropriation sociale de la nature) puis de l'UMR CNRS/ MNHN 7206 Eco-anthropologie et Ethnobiologie jusqu'à sa retraite, Aliette obtint l'honorariat au CNRS et le statut d'Attachée honoraire du Muséum, ce qui lui permit de poursuivre ses activités au CETMA, fondé 40 ans plus tôt. C'est en effet au CETMA qu'Aliette, en dehors des séjours d'enquête sur le terrain, a travaillé en permanence jusqu'à ses derniers jours. Le CETMA continue désormais de vivre dans l'esprit où Aliette l'a animé.

6 En 1971, François Beaudouin, Aliette Geistdoerfer et Bernard Koechlin, créèrent le Centre d'Ethno-Technologie en Milieux Aquatiques (C.E.T.M.A.) - afin de réunir ceux, chercheurs, étudiants, enseignants, professionnels et institutionnels, qui s'intéressent aux communautés maritimes, aux pêcheurs continentaux, à leurs pratiques d'exploitation des océans et des eaux intérieures.

7 Le CETMA a reçu le soutien du CNRS et du Muséum dès sa création. Il fut notamment parrainé par les professeurs Théodore Monod, directeur du laboratoire des Pêches Outremer du Muséum ${ }^{4}$, André Leroi-Gourhan et Michel Mollat. Joseph Gonella, directeur du laboratoire d'Océanographie physique du Muséum, mit à sa disposition des locaux du deuxième étage du 43 rue Cuvier ${ }^{5}$, où il demeure encore aujourd'hui.

C'est là que le CETMA gère et développe depuis sa fondation un centre de documentation scientifique pluridisciplinaire, sans équivalent en France, qui s'attache à la collection et à la diffusion des connaissances sur les populations vivant aux abords d'espaces aquatiques. Même si l'anthropologie y tient une place importante, Aliette a voulu que l'ensemble des disciplines et activités liées aux milieux aquatiques soient présentes, comme l'océanographie, la biologie marine ou encore les marines de pêche, marine marchande, militaire ou scientifique. L'ensemble constitue une source documentaire unique et homogène. D'abord gérée par l'équipe GDR-CNRS "Anthropologie maritime », la bibliothèque du CETMA est associée depuis 2010 par des conventions au MNHN et à la Direction des Bibliothèques. Elle fait partie du réseau de bibliothèques spécialisées de l'établissement.

Depuis sa création, la bibliothèque s'est enrichie d'ouvrages de différentes natures: dictionnaires spécialisés (noms de poisson dans différentes langues, glossaires, bibliographies...), monographies, numéros spéciaux thématiques de revues, actes de colloques, thèses, rapports, annuaires (de chercheurs, des formations de recherche, des musées, des centres de documentation, etc. $)^{6}$.

Ces ouvrages relèvent de disciplines diverses: anthropologie, sociologie, histoire, géographie, droit, sciences politiques, économie, écologie, océanographie, linguistique, sciences naturelles, arts et littérature. Des secteurs particuliers ont été privilégiés : les sociétés de marins-pêcheurs, les marins (commerce et militaire), les communautés littorales : pêcheurs à pied, conchyliculteurs, goémoniers, production halieutique, tenure maritime, techniques de pêche, économie des pêches, droit maritime, politique des pêches... Aucune aire géographique n'étant cependant privilégiée, ces ouvrages, articles et thèses traitent des sociétés maritimes du monde entier. 
11 Les thématiques couvertes par le centre de documentation du CETMA sont les suivantes :

- Les communautés de marins-pêcheurs, mais aussi marins de commerce et marins de guerre : vie familiale, femmes de marins, rémunérations, statut social et professionnel

- Les pêcheurs en eau douce et les bateliers

- Les métiers : apprentissage, formation, carrières ; le travail à bord et ses spécificités ; les notions de risques

- Les modes d'appropriation et de socialisation de la mer: zones de pêche, tenure maritime, accès privé, public à la mer et à ses ressources

- Les techniques de pêche au sens large, fabrication, réparation, artisanat, matériau, savoirs, les représentations des mondes marins

- Les techniques de navigation, embarcations et pratiques

- Les communautés littorales : pêcheurs à pied, conchyliculteurs, goémoniers, gardiens de phare

- Les institutions sociales, professionnelles et de formation

- Les politiques pêches et l'économie halieutique

- Les pratiques religieuses, rituelles et symboliques des gens de mer et des gens des eaux douce

- Les systèmes de représentation : parlers et langages, croyances, imaginaires marins

- Les métiers portuaires, le sauvetage, le transport maritime, la construction navale

- Les agents de distribution des produits marins : criées, mareyage, moyens de transport

- Les différents modes de transformation et consommation des produits marins

La bibliothèque est abonnée à plusieurs revues, bulletins périodiques, scientifiques et professionnels ${ }^{7}$.

13 Plusieurs ouvrages ont également été édités par le CETMA, sous la direction d'Aliette Geistdoerfer, notamment les Cahiers d'Anthropologie Maritime ( $\mathrm{N}^{\circ} 1$ à 5), Imagi-Mer et La Mer dévorée.

Deux principes ont guidé les membres du CETMA depuis sa création : participer, comme et avec le Muséum, à la construction d'une connaissance scientifique dans le domaine des relations homme/milieux aquatiques et contribuer à la diffuser auprès du plus grand nombre en collaborant notamment avec les milieux professionnels. Le CETMA accueille donc depuis toujours des chercheurs de différentes disciplines, doctorants et étudiants, journalistes, enseignants, professionnels de la mer, etc. Le CETMA reçoit par ailleurs également des groupes scolaires dans le cadre de la Fête de la Science à laquelle il participe chaque année.

15 Aujourd'hui, la bibliothèque est emblématique de l'étude pionnière des relations hommeenvironnement engagée au Muséum national d'Histoire naturelle. Elle est aussi un témoignage remarquable de l'évolution des problématisations des relations hommesmilieux aquatiques (vulnérabilité écologique des milieux et des conséquences sur les populations humaines, évolution des niveaux de productions halieutiques, changements climatiques, modifications des techniques et outils, etc.). A l'heure de la mise en place de nouvelles formes de gouvernance des ressources naturelles, particulièrement aquatiques, le centre de documentation du CETMA-Anthropologie maritime permet une mise en perspective, sur les 40 dernières années, des politiques de gestion de ces ressources aux échelles locales, régionales, nationales et internationales. 

nature, le centre de documentation du CETMA-Anthropologie maritime, demeure unique en son genre à l'échelon de la France et au-delà de ses frontières.

\section{NOTES}

1. Devenu «CETMA-Anthropologie Maritime».

2. Commission « Anthropologie, préhistoire, ethnologie ».

3. 3 Soutenue en 1981 à l'Université René-Descartes-Paris V-Sorbonne La pêche côtière aux Îles de la Madeleine: Québec.

4. Devenu laboratoire «Ichtyologie générale et appliquée ».

5. À partir de 2008, le CETMA rejoignit le premier étage de ce bâtiment, où il est toujours, non loin de ce qui était la bibliothèque d'Ethnobiologie-biogéographie.

6. Près de 4000 monographies cataloguées.

7. Près de 72 périodiques répertoriés.

\section{AUTEURS}

\section{ÉMILIE MARIAT-ROY}

Docteur de l'EHESS, membre associé du laboratoire IRD/MNHN PALOC et du CETMA-

Anthropologie maritime

\section{CHRISTOPHE SÉCULA}

Docteur du Muséum national d'Histoire naturelle, associé au CETMA-Anthropologie maritime 